\title{
Cooling Metals via Gap Plasmon Resonance
}

Jin-Woo Cho, Sung-Jun Park, Su-Jin Park, Young-Bin Kim, Yoon-Jong Moon, and SunKyung Kim*

Department of Applied Physics, Kyung Hee University, Gyeonggi-do 17104, Republic of Korea

This PDF file includes the following:

Supplementary Figures S1-S9

${ }^{*}$ Corresponding author: S.-K.K. (sunkim@khu.ac.kr) 


\section{Methods}

Fabrication of $\mathrm{Cu} / \mathrm{ZnS} / \mathrm{Cu}$ multi-cavity array. $\mathrm{Cu} / \mathrm{ZnS} / \mathrm{Cu}$ multi-cavity arrays were fabricated using an i-line stepper (I9C, Nikon) on a 6-inch Si substrate. A 200-nm-thick Cu reflector with a 20-nm-thick Ti adhesion layer was deposited on the Si substrates using electron beam evaporation (UEE, ULTEC). A 500-nm-thick ZnS dielectric spacer was deposited on the $\mathrm{Cu}$ reflector using the same electron beam evaporation. A 5- $\mu \mathrm{m}$-thick positive photoresist (AZ 4620) was coated on the $\mathrm{ZnS}$ layer for the photolithography process. Subsequently, a 50-nmthick $\mathrm{Cu}$ layer was deposited on the patterned photoresist using the same electron beam evaporation. An acetone solution (99\%, Sigma Aldrich) was used for the lift-off process, and an upper $\mathrm{Cu}$ grating was formed.

Optical characterization. Optical characterization of the fabricated $\mathrm{Cu} / \mathrm{ZnS} / \mathrm{Cu}$ multi-cavity arrays was performed using spectrometers and thermography cameras. For solar radiation wavelengths (0.3-2.5 $\mu \mathrm{m})$, a spectrophotometer (Cary5000, Thermo Fisher) equipped with an integrating sphere was used. An FTIR spectrometer (INVENIO R, Bruker) equipped with a gold-diffuser-coated integrating sphere (A562-G/Q, Thorlabs) and mercury telluride detector was used to obtain the absorptivity spectrum at thermal radiation wavelengths $(5-15 \mu \mathrm{m})$. For direct measurement of the emissivity spectrum, the same FTIR spectrometer equipped with a heater adapter, including a temperature controller (A540, Bruker), was used. For the emissivity measurement, each sample was heated at $50{ }^{\circ} \mathrm{C}$, and a black soot sample $\left(\varepsilon_{\text {avg }}=\right.$ 0.92) was used as a blackbody reference. To obtain the angle-resolved absorptivity spectrum, the same FTIR spectrometer equipped with a variable angle reflection accessory (Seagull, Harrick) was used. To capture the thermographic image of each sample, a short- $(3-5 \mu \mathrm{m}$, SC6000, FLIR) or long-wavelength (7.5-14 $\mu \mathrm{m}$, A655SC, FLIR) infrared camera was used, 
depending on the sample temperature. The average emissivity ( $\left.\varepsilon_{\text {avg }}\right)$ integrated 5-15 $\mu \mathrm{m}$, and weighted by the $330-\mathrm{K}$ blackbody spectra, was defined as follows:

$$
\varepsilon_{\mathrm{avg}}=\frac{\int_{5}^{15} \varepsilon(\lambda) I_{B B}(T, \lambda) d \lambda}{\int_{5}^{15} I_{B B}(T, \lambda) d \lambda}
$$

The effective emissivity ( $\varepsilon_{\text {eff }}$ ) was defined in the same way for a spectral range $8-13 \mu \mathrm{m}$. In addition, spectral radiance of a blackbody at temperature $T$ is

$$
I_{B B}(\mathrm{~T}, \lambda)=\frac{2 h c^{2}}{\lambda^{5}} \frac{1}{e^{h c /\left(\lambda k_{B} T\right)}-1}
$$

where $h$ is Planck's constant, $k_{b}$ is the Boltzmann constant, $c$ is the speed of light, and $\lambda$ is the wavelength. The average solar reflectivity, $R_{\text {avg }}$ was weighted by the AM $1.5 \mathrm{G}$ spectrum $\left(I_{A M 1.5 G}(\lambda)\right) 0.3-2.5 \mu \mathrm{m}$ was defined as follows:

$$
R_{\mathrm{avg}}=\frac{\int_{0.3}^{2.5} R(\lambda) I_{A M .15 G}(\lambda) d \lambda}{\int_{0.3}^{2.5} I_{A M .15 G}(\lambda) d \lambda},
$$

where $R(\lambda)$ denotes the measured reflectivity at a specific wavelength $(\lambda)$.

Electromagnetic Simulations. Electromagnetic simulations were conducted using finitedifference time-domain simulations (FDTD Solutions, Lumerical). The optical constants of the $\mathrm{Cu}, \mathrm{Ag}$, and $\mathrm{Au}$ materials used were derived from previous studies, ${ }^{43,44}$ depending on the considered wavelengths. The $\mathrm{ZnS}$ was treated as a non-dispersive material with a constant refractive index of 2.2 at all the considered wavelengths. ${ }^{40}$ A broadband, normally incident plane wave was used, and a frequency-domain power monitor was located to acquire reflectance. Periodic boundary conditions were employed along the $x$ - and $y$-directions, whereas perfectly matched layers were applied at the top and bottom boundaries, respectively. The spatial resolution was set to $1 \mathrm{~nm}$ along all the directions. Rigorous 
coupled-wave analysis software (DiffractMOD, Rsoft) simulations were used to obtain the angle-resolved absorptivity spectra of the multi-cavity arrays.

Outdoor cooling experiments. Outdoor cooling experiments were performed on the rooftop of the Applied Physics Department building in Kyung Hee University. The measurement system was composed of an acrylic box coated with an aluminized mylar film to minimize conduction heat flow from the ground. Each sample was contained in an acrylic container wrapped with an aluminized mylar film, except for the face of the solar illumination. A polystyrene thermal insulator was introduced on the backside of each sample holder. A midinfrared-transparent low-density polyethylene film was used as the windshield. Temporal changes in temperature were obtained using adhesive thermocouples (SA1-K-72-SC, Omega engineering) attached to the Si substrates and recorded with a data logger (RDXL12SD, Omega Engineering) every $10 \mathrm{~s}$. For comparison, a planar $\mathrm{Cu} / \mathrm{ZnS} / \mathrm{Cu}$ multilayer exhibiting the same average solar reflectivity was prepared.

Heat-transfer simulations. Heat transfer simulations were performed to support the results of the outdoor cooling experiments. The steady-state temperatures $\left(T_{s}\right)$ were determined using the following relation:

$$
P_{\text {rad }}(T)-P_{a t m}\left(T_{a t m}\right)-P_{\text {solar }}+P_{\text {cond }+ \text { conv }}=0,
$$

where $P_{\text {rad }}(T), P_{a t m}\left(T_{a t m}\right), P_{\text {solar }}$, and $P_{\text {cond }+ \text { conv }}$ are the thermal radiation energy from the multi-cavity array, thermal radiation energy from the atmosphere, solar absorption, and non-radiative heat transfer from the sample via conduction and convection, respectively. The detailed definition of each parameter is as follows:

$$
P_{r a d}(T)=\int d \Omega \cos \theta \int_{0}^{\infty} d \lambda I_{B B}(T, \lambda) \varepsilon(\lambda, \theta),
$$


where $\int d \Omega=2 \pi \int_{0}^{\pi / 2} d \theta \sin \theta \int_{0}^{2 \pi} d \phi$ denotes the angular integral over a hemisphere, and $\varepsilon(\lambda, \theta)$ denotes the sample spectral and angular emissivity. Additionally,

$$
P_{a t m}\left(T_{a t m}\right)=\int d \Omega \cos \theta \int_{0}^{\infty} d \lambda I_{B B}(T, \lambda) \varepsilon(\lambda, \theta) \varepsilon_{a t m}(\lambda, \theta),
$$

where $I_{B B}\left(T_{a t m}, \lambda\right)$ denotes the spectral radiance of a blackbody at atmospheric temperature, and $\varepsilon_{\text {atm }}(\lambda, \theta)$ denotes the atmospheric emissivity. ${ }^{42}$ In addition,

$$
P_{\text {solar }}=\int_{0}^{\infty} d \lambda A_{\text {avg }} I_{A M 1.5 G}(\lambda)
$$

where $I_{A m 1.5 G}(\lambda)$ denotes the measured solar irradiance $\left(I_{\text {sun }}\right)$, and $A_{\text {avg }}\left(=1-R_{\text {avg }}\right)$ denotes the measured absorptivity.

$$
P_{\text {cond }+ \text { conv }}=h_{c}\left(T-T_{a t m}\right)
$$

where $h_{c}$ denotes the combined non-radiative heat transfer coefficient, including the conductive $\left(h_{\text {cond }}\right)$ and convective $\left(h_{\text {conv }}\right)$ processes. 
a

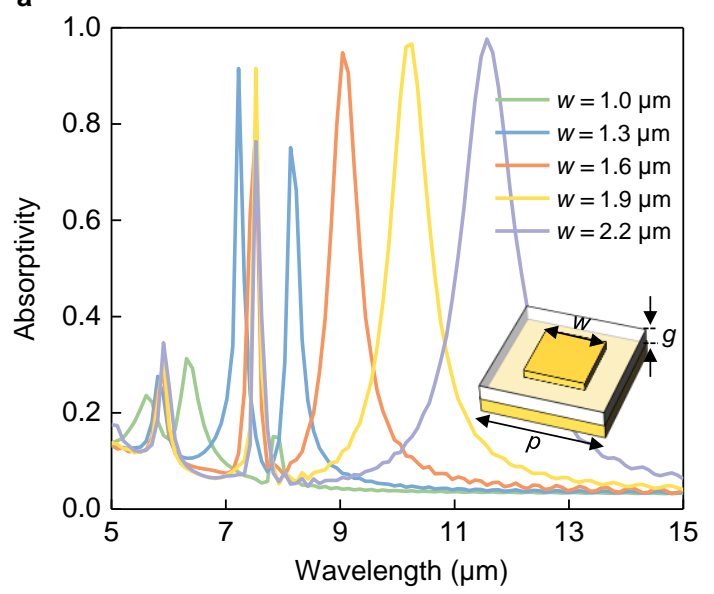

b

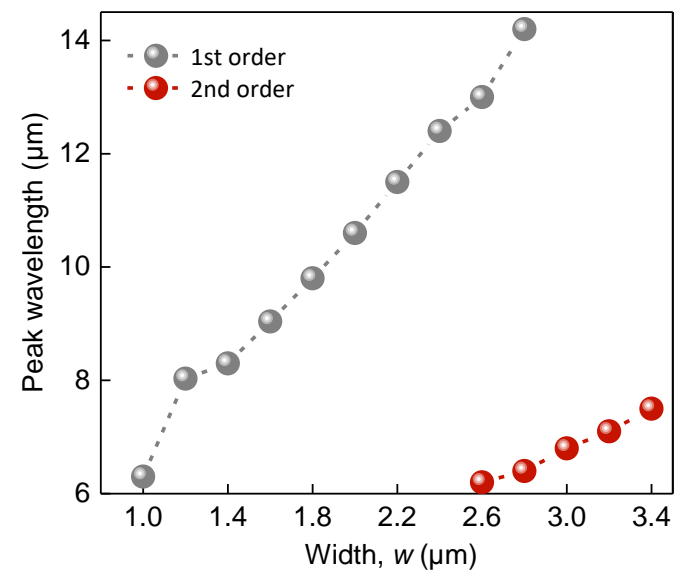

Figure S1. Absorptivity spectra of single-cavity arrays depending on the width $(w)$ of the upper metal grating. (a) Simulated absorptivity spectra of $\mathrm{Cu} / \mathrm{ZnS} / \mathrm{Cu}$ single-cavity arrays with $w=1.0,1.3,1.6,1.9$, and $2.2 \mu \mathrm{m}$, respectively. The pitch $(p)$ and dielectric spacer thickness $(\mathrm{g})$ are fixed at $7.2 \mu \mathrm{m}$ and $500 \mathrm{~nm}$, respectively. (Inset) Schematic of the structural parameters. (b) Simulated absorption peak wavelengths of $\mathrm{Cu} / \mathrm{ZnS} / \mathrm{Cu}$ single-cavity arrays as a function of $w$. Two different groups result from gap plasmon cavity modes with different orders. The data point at $w \sim 1.2 \mu \mathrm{m}$ deviates from the linear trend because of the coupling with the first-order diffraction mode (i.e., the anticrossing effect). 

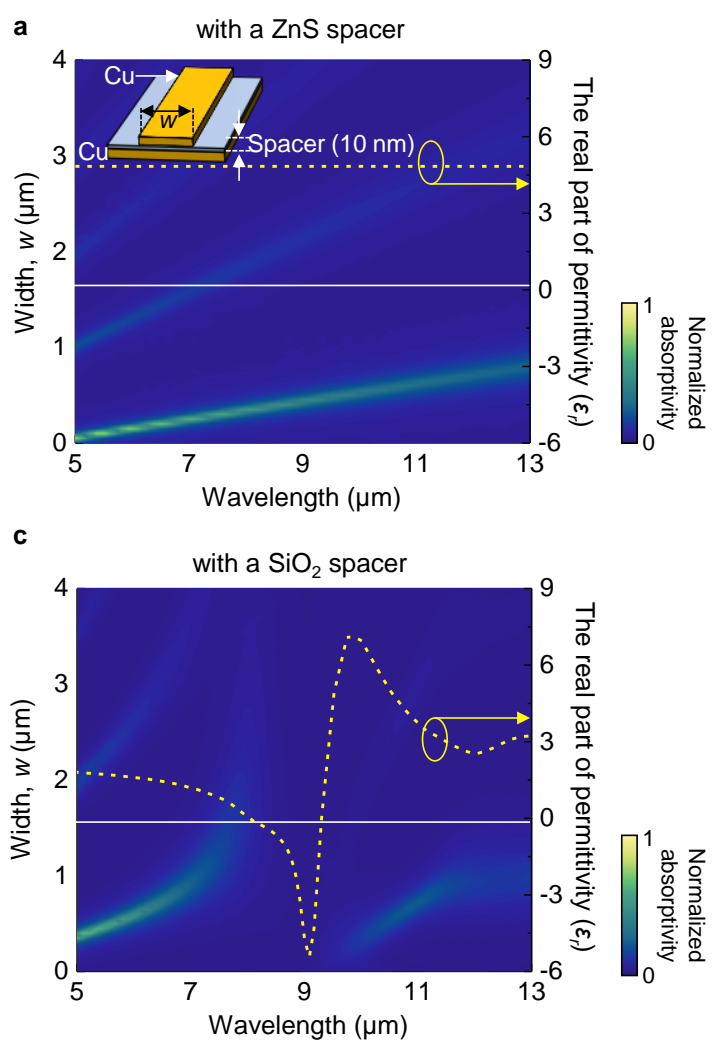
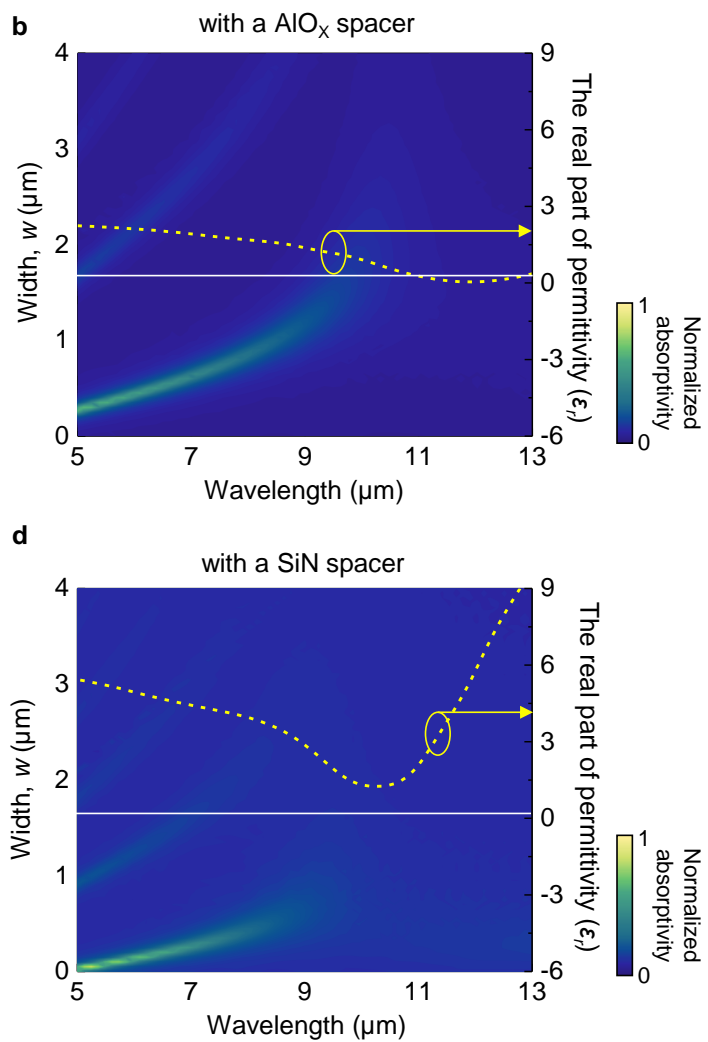

Figure S2. Absorptivity spectra of single-cavity arrays with various dielectric spacer materials. (a-d) Simulated normalized absorptivity depending on the width $(w)$ of the upper $\mathrm{Cu}$ grating for various dielectric spacer materials: $\mathrm{ZnS}(\mathrm{a}), \mathrm{AlOx}(\mathrm{b}), \mathrm{SiO}_{2}$ (c), and $\mathrm{SiN}$ (d). The white solid line indicates that the real part of permittivity $\left(\varepsilon_{\mathrm{r}}\right)$ equals 0 . In each plot, the most intense band is identified as the fundamental gap plasmon resonance. Only for the $\mathrm{ZnS}$ dielectric spacer, the fundamental gap plasmon resonance is continuously shifted to longer wavelengths with increasing $w$. In comparison, for the other dielectric spacers, the fundamental gap plasmon resonance diminishes near the wavelength of $\varepsilon_{\mathrm{r}}=0$ (i.e., an epsilon-near-zero wavelength). For these simulations, one-dimensional $\mathrm{Cu} / \mathrm{dielectric} / \mathrm{Cu}$ single-cavity arrays are considered. 


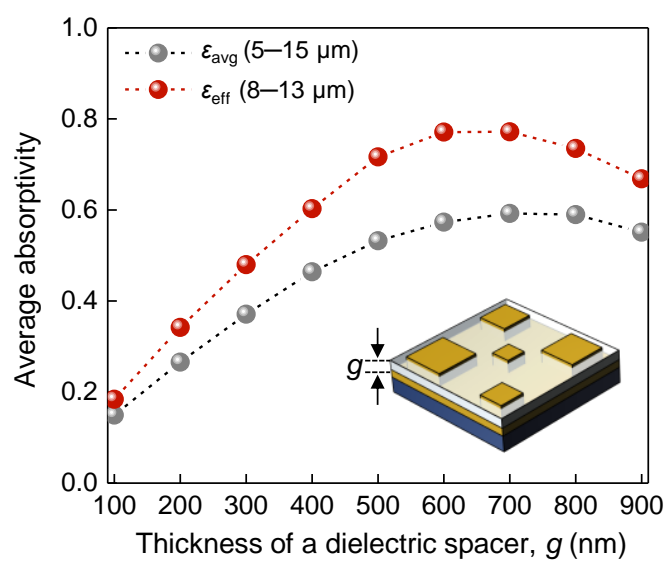

Figure S3. Optimum thickness $(g)$ of a dielectric spacer $(\mathrm{ZnS})$ for the critical coupling condition. The average ( $\left.\varepsilon_{\text {avg }}\right)$ and effective ( $\left.\varepsilon_{\text {eff }}\right)$ emissivity values of the $\mathrm{Cu} / \mathrm{ZnS} / \mathrm{Cu}$ multicavity array in Figure 2, weighted by the $330-\mathrm{K}$ blackbody spectrum, are calculated at various $g$ values. (Inset) Schematic of the simulated structure. 

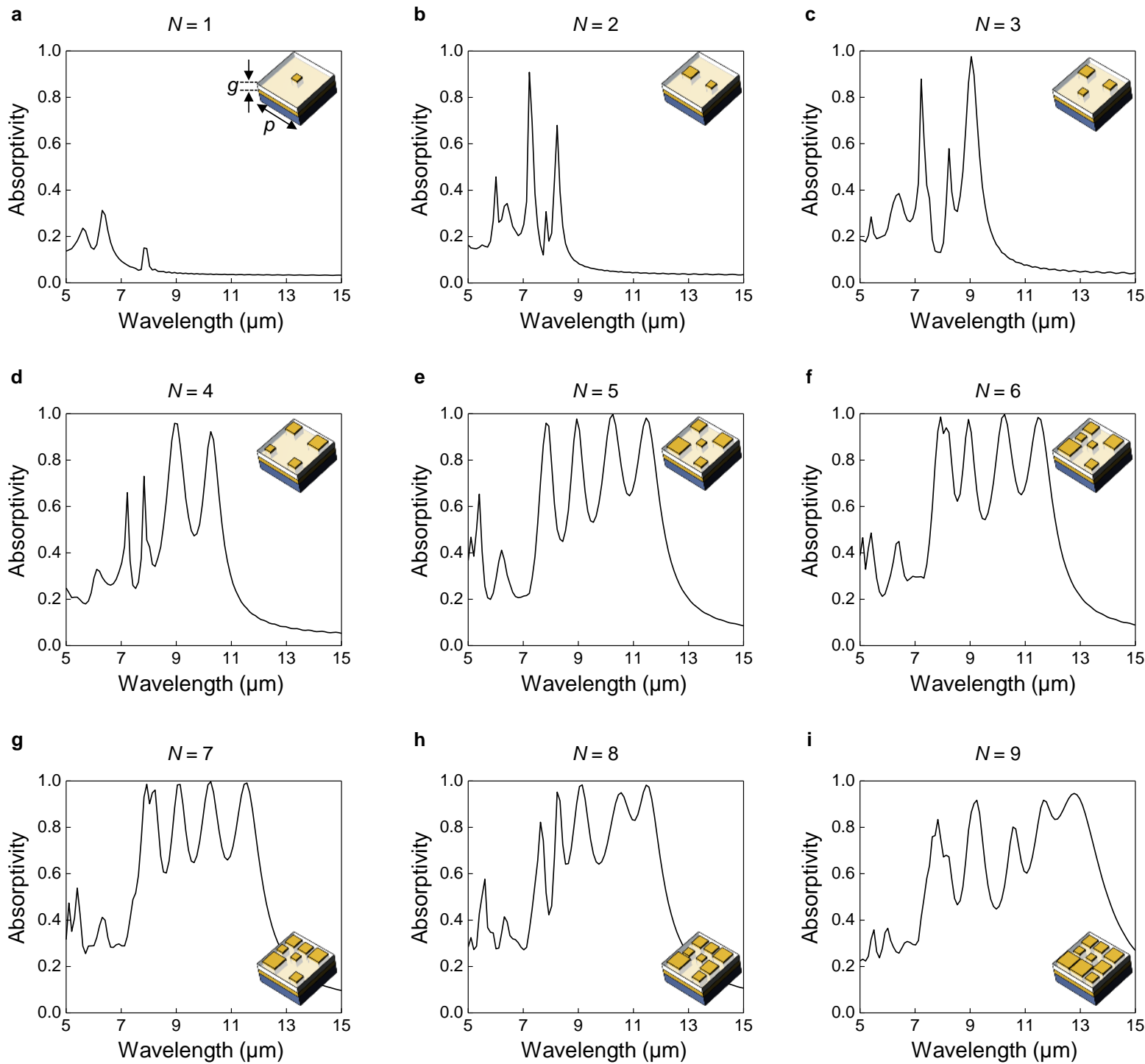

Figure S4. Absorptivity spectra of $\mathrm{Cu} / \mathrm{ZnS} / \mathrm{Cu}$ multi-cavity arrays depending on the number $(N)$ of cavities. (a-i) Simulated absorptivity spectra of $\mathrm{Cu} / \mathrm{ZnS} / \mathrm{Cu}$ multi-cavity arrays with different $N$ values. The width $(w)$ of the cavity added to the former structure increases from 1.15 to $2.2 \mu \mathrm{m}$ with a constant step of $0.15 \mu \mathrm{m}$. For $N=1, w=1 \mu \mathrm{m}$. For all the simulated structures, the pitch $(p)$ and dielectric spacer thickness $(g)$ are fixed at $7.2 \mu \mathrm{m}$ and $500 \mathrm{~nm}$, respectively. (Inset) Schematic of each simulated structure. 

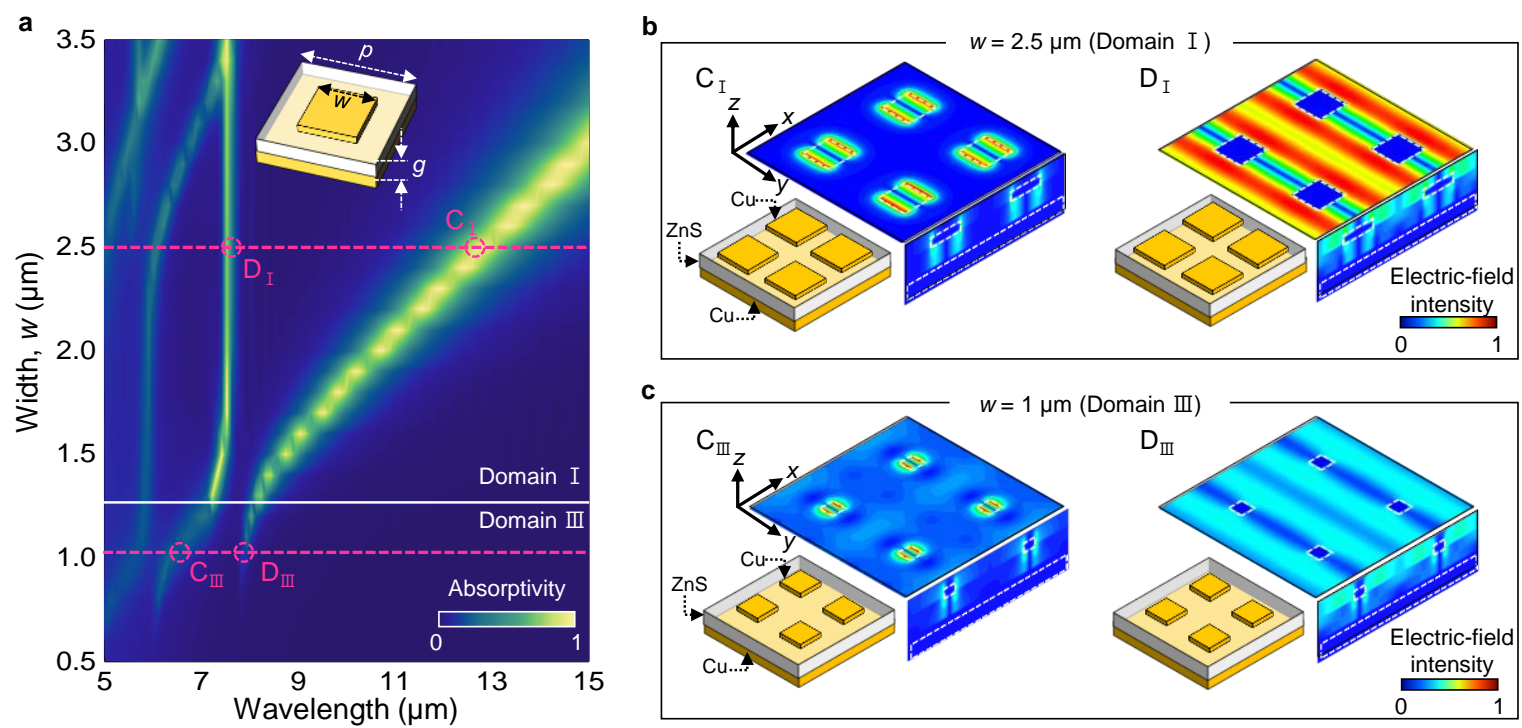

Figure S5. Photonic band dispersion of a $\mathrm{Cu} / \mathrm{ZnS} / \mathrm{Cu}$ single-cavity array. (a) Surface plot depicting simulated absorptivity spectra of $\mathrm{Cu} / \mathrm{ZnS} / \mathrm{Cu}$ single-cavity arrays with a fixed pitch $(p)$ of $7.2 \mu \mathrm{m}$, varying its width $(w)$. The surface plot is divided into Domains I and III (see Figure $3 b$ ). The dashed circles indicate the gap plasmon cavity modes and diffraction modes, as labelled $\mathrm{C}_{\mathrm{I}}, \mathrm{C}_{\mathrm{III}}, \mathrm{D}_{\mathrm{I}}$, and $\mathrm{D}_{\text {IIII, }}$ respectively. In Domain I, the gap plasmon cavity modes and diffraction modes are clearly separated. With increasing $w$, the gap plasmon cavity modes are linearly redshifted while the diffract modes are fixed at the same wavelength. In Domain III, when $w$ is smaller than $1.4 \mu \mathrm{m}$, the mode anticrossing behavior is observed. (Inset) Schematic of the simulated structure. (b,c) Distribution of electric-field intensity for the gap plasmon cavity modes and diffraction modes as labelled $\mathrm{C}_{\mathrm{I}}, \mathrm{C}_{\mathrm{III}}, \mathrm{D}_{\mathrm{I}}$, and $\mathrm{D}_{\mathrm{III}}$ in (a), respectively. For all the simulated structures, the pitch $(p)$ and dielectric spacer thickness $(g)$ are fixed at $7.2 \mu \mathrm{m}$ and $500 \mathrm{~nm}$, respectively. 


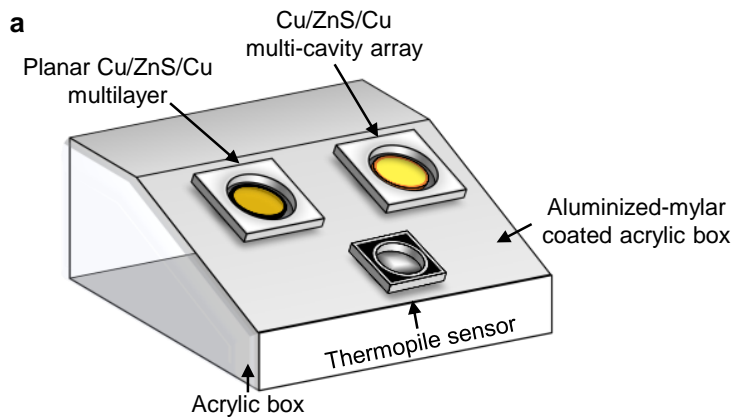

b

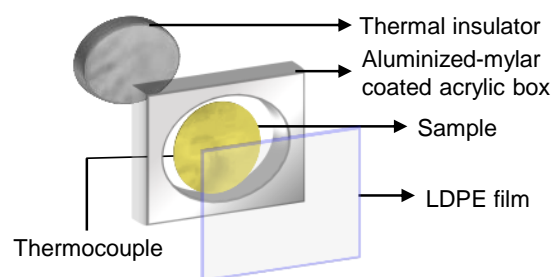

Figure S6. Outdoor cooling measurement setup. (a,b) Schematics illustrating outdoor cooling measurement setup (a) and its sample holder (b). 


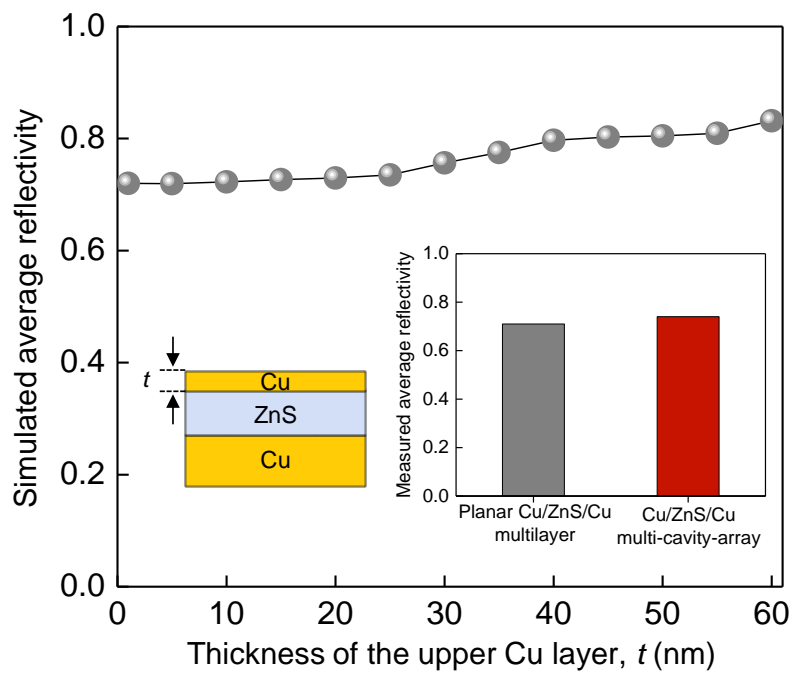

Figure S7. Solar reflectivity of planar $\mathrm{Cu} / \mathrm{ZnS} / \mathrm{Cu}$ multilayers. Simulated average solar (0.3$2.5 \mu \mathrm{m})$ reflectivity depending on the thickness $(t)$ of the upper Cu layer. (Insets) Schematic of the simulated structure (left) and measured average solar reflectivity of the planar $\mathrm{Cu} / \mathrm{ZnS} / \mathrm{Cu}(10 / 500 / 200 \mathrm{~nm})$ multilayer and $\mathrm{Cu} / \mathrm{ZnS} / \mathrm{Cu}(50 / 500 / 200 \mathrm{~nm})$ multi-cavity array in Figures 2 and 4 (right). 

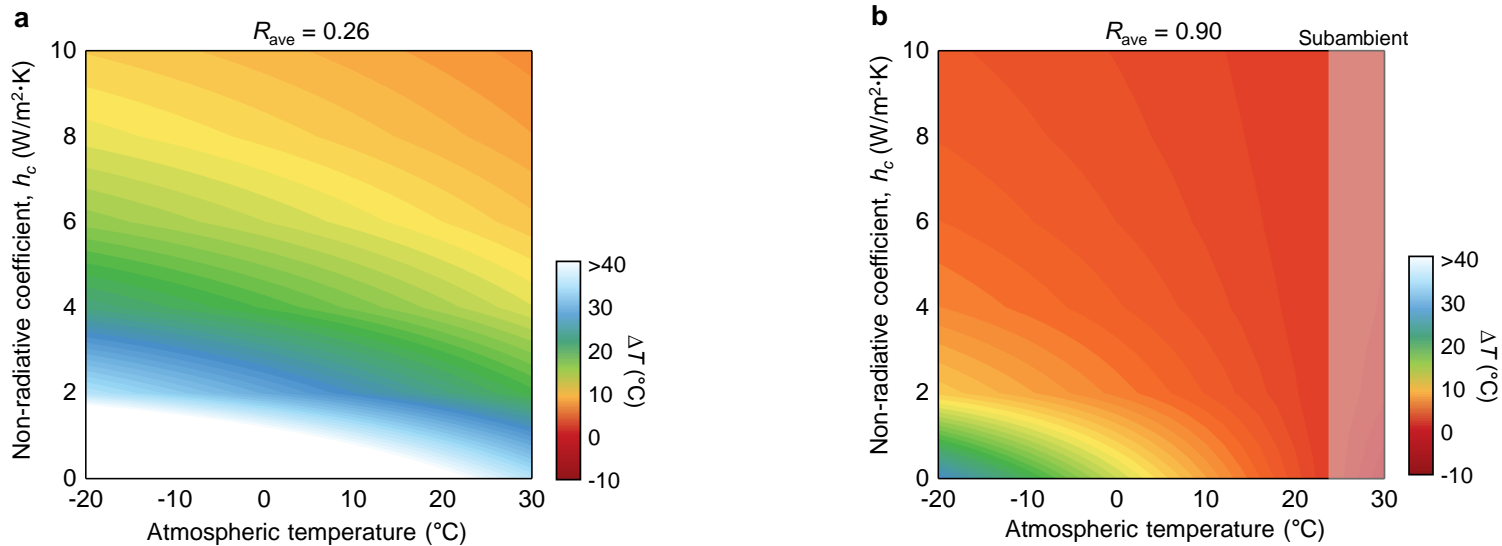

Figure S8. Performance of radiative coolers depending on their solar reflectivity. (a,b) Calculated difference $(\Delta T)$ between the steady-state temperature $\left(T_{\mathrm{s}}\right)$ of a radiative cooler and atmospheric temperature $\left(T_{\mathrm{atm}}\right)$ as functions of $T_{\mathrm{atm}}$ and non-radiative coefficient $\left(h_{\mathrm{c}}\right)$ when the solar reflectivity is 0.74 (a) or 0.90 (b). The emissivity of the simulated radiative cooler is the same as that of our $\mathrm{Cu} / \mathrm{ZnS} / \mathrm{Cu}$ multi-cavity array. The input solar irradiance is one-sun intensity. In the case of (a), which is identical to our multi-cavity array, the considerable solar energy absorption hinders the achievement of subambient cooling $\left(\Delta T<0^{\circ} \mathrm{C}\right)$ during daytime. However, when the solar reflectance is $90 \%$, such subambient cooling appears at $T_{\text {atm }}>24{ }^{\circ} \mathrm{C}$ (shaded area). 


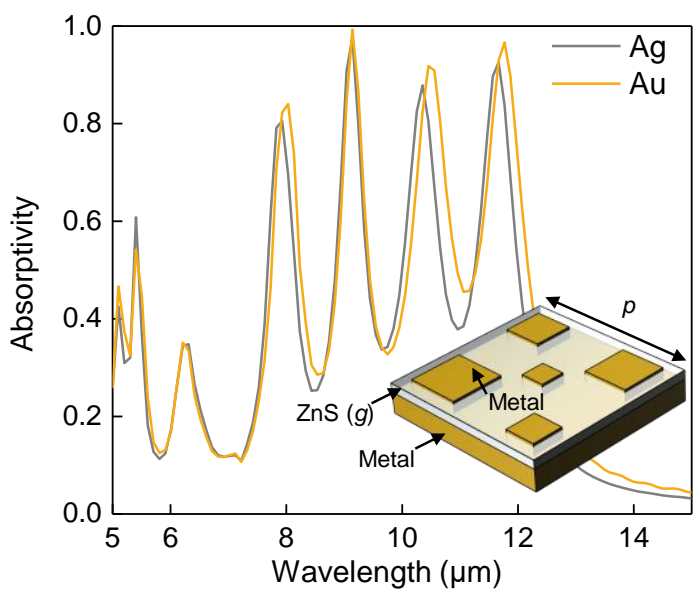

Figure S9. Gap plasmon resonance with other metals. Simulated absorptivity spectra of metal/ZnS/metal multi-cavity arrays with different metal materials (Ag and $\mathrm{Au}$ ). (Inset) Schematic of the simulated structure parameter. The pitch $(p)$ and dielectric spacer thickness $(g)$ are fixed at $7.2 \mu \mathrm{m}$ and $500 \mathrm{~nm}$, respectively. 